\title{
Efeito do Peso Inicial dos Pintos e do Período da Dieta Pré-Inicial sobre o Desempenho de Frangos de Corte
}

\author{
José Henrique Stringhini ${ }^{1,6}$, Aletéia di Resende ${ }^{2}$, Marcos Barcellos Café ${ }^{3}$, Nadja Susana \\ Mogyca Leandro ${ }^{4}$, Maria Auxiliadora Andrade 5
}

\begin{abstract}
RESUMO - O presente trabalho foi conduzido com o objetivo de avaliar o uso de dietas pré-iniciais, por diferentes períodos, para frangos de corte criados a partir de pintos com diferentes pesos ao primeiro dia. No aviário experimental da Escola de Veterinária da UFG, 280 pintos de um dia foram distribuídos, em baterias aquecidas, em um delineamento inteiramente casualizado, com seis tratamentos e quatro repetições de doze aves, em um esquema fatorial 2 x 3 (peso do pinto, maior e menor que 40g, e programas de fornecimento de ração pré-inicial, 1 a 7, 1 a 10 e 1 a 14 dias). O desempenho dos pintos não foi influenciado pelo período de fornecimento das dietas, mas o peso dos pintos no alojamento influenciou o consumo de ração das aves. Não foi observado efeito dos tratamentos no consumo de nutrientes para o período de fornecimento da dieta pré-inicial, mas os pintos com menor peso no alojamento proporcionaram menor consumo de nutrientes e de energia. Com base nos resultados obtidos, é possível concluir que peso do pinto inferior a $40 \mathrm{~g}$ influencia uma redução significativa no consumo e mostra uma tendência de pior peso dos frangos ao final do experimento. Os parâmetros morfométricos avaliados não foram afetados pelos tratamentos.
\end{abstract}

Palavras-chave: desempenho, frangos de corte, peso dos pintos de um dia, ração pré-inicial

\section{Effect of One-day Chicks Weight and Pre-starter Diets on Broiler Performance}

\begin{abstract}
This research was carried out to evaluate the use of pre-starter diets, through different periods to broilers with different first day weights. Two hundred and eighty one-day old chicks were allotted in brooded battery cages, in a completely randomized design, with six treatments and four replicates in a factorial design $2 \times 3$, (chicks weight - higher or lower than $40 \mathrm{~g}$, and pre-starter periods of feeding, 1 to 7; 1 to 10 and 1 to 14 days). The performance was not influenced by pre-starter diets periods, but the first-day weight of chicks reduced feed intake and live weight at 42 days of age. For energy and nutrient intake no effect was observed for pre-starter periods, but for the light chicks, the values of consumption were lower than heavier ones for all nutrients and energy. It is possible to conclude that chicks with less than 40 grams one-day weight influenced the reduction in feed intake and the weight gain. For the measures of the different relationship among the inside organs no differences were observed.
\end{abstract}

Key Words: broilers, pre-starter rations, one-day chicks weight, performance

\section{Introdução}

Os primeiros dias dos pintos de corte têm sido estudados por diferentes pesquisadores, tendo em vista seu ganho genético nos últimos anos, considerado crucial para determinar o bom desempenho do frango de corte moderno. Porém, com o aumento do alojamento de pintos de corte, ovos de matrizes novas têm sido incubados, o que concorre para uma produção de pintos de um dia com maior variação no seu peso inicial (Oliveira, 1981; Okada, 1994; Castro, 1996; Vieira \& Moran, 1998a,b).
Preconiza-se uma dieta diferenciada nos primeiros dias de vida das aves, porque neste período ocorre uma fisiologia digestiva peculiar, principalmente na primeira semana, pois as aves não estão plenamente adaptadas à digestão de carboidratos e lipídios (Serafin \& Nesheim, 1970; Moran, 1985; Dibner, 1996; Nir, 1998; Penz \& Vieira, 1998; Toledo et al., 2001). Ressalte-se que esses conhecimentos propiciaram o desenvolvimento de rações pré-iniciais.

Em estudos desenvolvidos sobre o tempo de troca destas dietas pré-iniciais para iniciais, foi observado que as aves são capazes de se adaptar a regimes

\footnotetext{
${ }^{1}$ Professor, Departamento de Produção Animal, EV-UFG, Caixa Postal 131, Departamento de Produção Animal, Escola de Veterinária da UFG, 74001-970, Goiânia, Goiás. E.mail: henrique@vet.ufg.br

2 Zootecnista, Especialista em Zootecnia, Departamento de Produção Animal, EV/UFG

3 Professor, Departamento de Produção Animal, EV-UFG. E.mail: mcafe@vet.ufg.br

4 Professor, Departamento de Produção Animal, EV-UFG. E.mail: mogyca@vet.ufg.br

5 Professor, Departamento de Medicina Veterinária, EV-UFG. E.mail: maa@vet.ufg.br

${ }^{6}$ Bolsista do CNPq.
} 
alimentares diferentes sem prejuízo de seu peso ao abate (Trindade et al., 1982), e o programa alimentar com quatro dietas foi o que proporcionou menor custo por quilograma de peso vivo e menor mortalidade (Franco et al., 1990; Shiroma et al., 1996). Já o tempo de fornecimento das dietas pré-iniciais por 7 ou 14 dias de idade não proporcionou diferenças de desempenho para frangos de corte (Franco et al., 1983; Zúniga et al., 1994; Shiroma et al., 1996; Araújo et al., 1999).

Segundo Nir (1998) e Penz \& Vieira (1998), manter a dieta pré-inicial por períodos superiores a sete dias compromete seus benefícios, visto que a partir desse período as aves já apresentam uma boa adaptação anatomofisiológica do seu aparelho digestório.

Assim considerando que ainda são escassas as informações sobre o efeito desses programas alimentares para pintos de corte com diferentes pesos ao primeiro dia, desenvolveu-se este trabalho, que teve como objetivo verificar o desempenho produtivo de frangos de corte criados a partir de pintos com diferentes pesos no primeiro dia de idade, recebendo dietas pré-iniciais por também diferentes períodos.

\section{Material e Métodos}

Este trabalho foi conduzido no Aviário Experimental do Departamento de Produção Animal da Escola de Veterinária da Universidade Federal de Goiás entre os meses de outubro e novembro de 1997.

Foram selecionados 280 pintos da linhagem Indian River x Hubbard, sexados e vacinados contra Marek no incubatório. As aves foram criadas em baterias aquecidas até 21 dias e transferidas para boxes de 1,0 x $1,0 \mathrm{~m}$, entre o $22^{\circ}$ e o $42^{\circ}$ dias de vida. Foram utilizadas três baterias de aço galvanizado, de cinco andares, com divisões de $0,33 \times 0,50 \mathrm{~m}$ e divididas ao meio, somando 24 unidades experimentais, equipadas com comedouros e bebedouros tipo lineares (calha). Foi utilizada uma lâmpada incandescente de $100 \mathrm{~W}$ para cada andar, até aproximadamente 12 dias de idade, para aquecimento das aves. O manejo até 21 dias incluiu a limpeza diária dos bebedouros e a troca de água e abastecimento de comedouros duas vezes ao dia. Aos 21 dias de idade as aves foram transferidas para boxes em piso, que se encontravam forrados com cepilho de madeira e equipados com bebedouros lineares de água corrente (calha) e comedouros tubulares. No $7 \stackrel{\mathrm{o}}{ }, 10^{\circ}, 14^{\circ}, 21^{\circ} \stackrel{\circ}{ }, 35^{\circ} \mathrm{o}$ e $42^{\circ} \underline{\mathrm{o}}$ dia de idade, as aves foram pesadas e foram calculados o consumo de ração e a conversão alimentar.

Foi utilizado o delineamento inteiramente casualizado em um esquema fatorial 2 × 3 (dois pesos do pinto - acima e abaixo de $40 \mathrm{~g}$-, e três programas de fornecimento de dieta pré-inicial, 1 a 7,1 a 10 e 1 a 14), totalizando seis tratamentos com quatro repetições de doze aves cada um.

As rações pré-iniciais foram formuladas à base de milho e farelo de soja, e fornecidas às aves até os 14 dias de idade. A partir da fase pré-inicial, forneceram-se uma dieta inicial até os 21 dias, uma dieta de crescimento, até os 35 dias, e uma ração final, até os 42 dias de idade. As fórmulas das rações utilizadas e os valores de suas composições percentuais são apresentadas na Tabela 1. Avaliaram-se o ganho de peso, o consumo de ração, a conversão alimentar, a viabilidade e o fator de produção (FP), calculado pela seguinte fórmula:

$\mathrm{FP}=[($ ganho de peso $x$ viabilidade em \%) $/($ idade em dias $x$ conversão alimentar)] / 10

Aos 21 e 42 dias de idade, uma ave por repetição foi sacrificada no Laboratório do Setor de Doenças de Aves do Departamento de Medicina Veterinária da UFG onde foi feita a pesagem individual e, depois de quatro horas de jejum, foram retirados o intestino vazio, o fígado, o pró-ventrículo, a moela, o coração, o pâncreas, o baço e a bursa de Fabricius, que foram pesados, medidos e avaliados como relação corporal ( $\mathrm{RC}=$ (peso do órgão/peso da ave)*100).

Os dados obtidos no experimento foram submetidos à análise de variância e foio aplicado o teste de Tukey (5\%). 
Tabela 1 - Composição e níveis nutricionais das rações experimentais

Table 1 - Percentual and nutritional composition of the experimental rations

\begin{tabular}{|c|c|c|c|c|}
\hline $\begin{array}{l}\text { Ingrediente }(\%) \\
\text { Ingredient }\end{array}$ & $\begin{array}{c}\text { Pré-inicial } \\
\quad(1-14 \mathrm{~d}) \\
\text { Pre-starter }\end{array}$ & $\begin{array}{c}\text { Inicial } \\
\text { (14-21d) } \\
\text { Starter }\end{array}$ & $\begin{array}{c}\text { Crescimento } \\
\text { (21-35d) } \\
\text { Grower }\end{array}$ & $\begin{array}{c}\text { Final } \\
(35-42 d) \\
\text { Finishing }\end{array}$ \\
\hline Milho (Corn) & 60,30 & 63,81 & 62,50 & 65,31 \\
\hline Farelo de gérmen de milho (Corn germ meal) & 0 & 0 & 10,00 & 15,00 \\
\hline Farelo de soja (Soybean meal) & 30,8 & 20,80 & 9,30 & 7,10 \\
\hline Soja integral (Fullfat soybean) & 2,7 & 7,10 & 7,20 & 1,20 \\
\hline Farinha de carne $48 \%$ (Meat and bone meal 48\%) & 3,0 & 6,80 & 6,40 & 5,80 \\
\hline Farinha de sangue $80 \%$ (Blood meal $80 \%$ ) & 0 & 0 & 3,00 & 4,00 \\
\hline Sal (Salt) & 0,45 & 0,45 & 0,30 & 0,30 \\
\hline Calcário (Limestone) & 0,90 & 0,20 & 0,30 & 0,30 \\
\hline Fosfato bicálcico (Dicalcium phosphate) & 0,80 & 0 & 0 & 0 \\
\hline Bicarbonato de sódio (Sodium bicarbonate) & 0 & 0 & 0,15 & 0,15 \\
\hline DL-Metionina (DL-Methionine) & 0,21 & 0,21 & 0,18 & 0,13 \\
\hline L-Lisina $80 \%$ (L-Lysine $80 \%)$ & 0 & 0,03 & 0,01 & 0,03 \\
\hline Suplemento vitamínico ${ }^{1,2,3}$ (Vitamin supplement) & 0,06 & 0,05 & 0,05 & 0,05 \\
\hline Suplemento mineral ${ }^{4}$ (Mineral supplement) & 0,10 & 0,10 & 0,10 & 0,10 \\
\hline Veículo + aditivos (Inert and feed additives) & 0,68 & 0,45 & 0,51 & 0,53 \\
\hline \multicolumn{5}{|l|}{ Níveis nutricionais (Nutritional levels) } \\
\hline Proteína $(\%)$ (Protein ) & 21,96 & 21,03 & 19,90 & 17,98 \\
\hline Eneregia metabolizável (kcal/kg) & 2.938 & 3.036 & 3.094 & 3.110 \\
\hline \multicolumn{5}{|l|}{ Metabolizable energy $(\mathrm{kcal} / \mathrm{kg})$} \\
\hline Met+Cist (\%) (Methionine + Cystine) & 0,90 & 0,86 & 0,80 & 0,70 \\
\hline Metionina (\%) (Methionine) & 0,55 & 0,53 & 0,49 & 0,42 \\
\hline Lisina $(\%)$ (Lysine) & 1,174 & 1,105 & 1,052 & 0,948 \\
\hline Cálcio (\%) (Calcium) & 1,07 & 1,07 & 1,14 & 1,08 \\
\hline Fósforo disponível (\%) (Available phosphorus) & 0,41 & 0,51 & 0,52 & 0,50 \\
\hline
\end{tabular}

${ }_{1}^{1}$ Ração inicial: cloreto de colina $220 \mathrm{~g} / \mathrm{t}$ e suplemento vitamínico, níveis de garantia por kg de produto: 8.000.000 Ul vit. A, 2.000.000Ul vit. D3, 15.000 UI vit. E, $1.800 \mathrm{mg}$ vit. K, $1.800 \mathrm{mg}$ vit. B1, $6.000 \mathrm{mg}$ vit. B2, $2.800 \mathrm{mg}$ vit. B6, 12.000 mcg vit. B12, $40.000 \mathrm{mg}$ niacina, $1.000 \mathrm{mg}$ ácido fólico, $15.000 \mathrm{mg}$ ácido pantotênico, $60 \mathrm{mg}$ de biotina, $300 \mathrm{mg}$ selênio, $30 \mathrm{~g}$ antioxidante;

2 Ração de crescimento: cloreto de colina $286 \mathrm{~g} / \mathrm{t}$ e suplemento vitamínico, níveis de garantia por kg de produto: $7.000 .000 \mathrm{Ul}$ vit. A, $1.500 .000 \mathrm{UI}$ vit. D3, $12.000 \mathrm{UI}$ vit. E, $1.500 \mathrm{ng}$ vit. K, $1.600 \mathrm{mg}$ vit. B1, $5.000 \mathrm{mg}$ vit. B2, $2.600 \mathrm{mg}$ vit. B6, $10.000 \mathrm{mcg}$ vit. b12, 35.000 $\mathrm{mg}$, niacina, $700 \mathrm{mg}$ ácido fólico, $13.000 \mathrm{mg}$, ácido pantotênico, $300 \mathrm{mg}$ selênio, $20 \mathrm{~g}$ antioxidante;

${ }^{3}$ Ração final: cloreto de colina $280 \mathrm{~g} / \mathrm{t}$ + frango abate concentrado - suplemento vitamínico, níveis de garantia por kg de produto: $2.500 .000 \mathrm{UI}$ vit. A, $700.000 \mathrm{UI}$ vit. D3, $7.000 \mathrm{Ul}$ vit. E, $700 \mathrm{mg}$ vit. K, $2.500 \mathrm{mg}$ vit. B1, $2.500 \mathrm{mg}$ vit. B2, $6.000 \mathrm{mcg}$ vit. B12, $26.000 \mathrm{mg}$ niacina, 9.000 $\mathrm{mg}$ ácido pantotênico, $200 \mathrm{mg}$ selênio, $20 \mathrm{~g}$ antioxidante;

${ }^{4}$ Para todas as rações: suplemento mineral, níveis de garantia por kg de produto: $150.000 \mathrm{mg}$ manganês, $100.000 \mathrm{mg}$ zinco, $100.000 \mathrm{mg}$ ferro, $16.000 \mathrm{mg}$ cobre, $1.500 \mathrm{mg}$ iodo.

${ }^{5}$ Para a ração inicial: ácido propiônico (interprop), $1 \mathrm{~kg} / \mathrm{t}$, antioxidante (bht), $150 \mathrm{~g} / \mathrm{t}$, (monteban) + nicarbazina, $700 \mathrm{~g} / \mathrm{t}, \mathrm{sulfato}$ de cobre + violeta de genciana (micogen), $100 \mathrm{~g} / \mathrm{t}$; olaquindox $300 \mathrm{~g} / \mathrm{t}$.

${ }^{6}$ Para a ração de crescimento: ácido 3-nitro 35 g/t, ácido propiônico (interprop), 500 g/t, antioxidante (bht), 150 g/t, coxistac, 550 g/t.

\section{Resultados e Discussão}

Os pesos obtidos para os pintos no primeiro dia foram 36,4 e 47,5 g, respectivamente, para os abaixo e acima de 40 gramas. Não foi observada interação significativa $(P>0,05)$ entre os fatores estudados.

$\mathrm{Na}$ última semana, o resultado de conversão alimentar mostrou uma variação não explicável para as aves que receberam dieta pré-inicial de 1 a 10 dias, sendo superior àquelas com períodos de 1 a 7 e 1 a 14 da mesma ração (Tabela 2). Os demais resultados não foram afetados pelo programa alimentar (Tabela 2).

$\mathrm{O}$ peso do pinto no primeiro dia $(\mathrm{P}>0,05)$ influenciou o peso dos frangos aos 35 dias de idade (Tabela 3). Embora a diferença não tenha sido significativa aos
42 dias, foi observado que pintos mais leves ao primeiro dia resultaram em frangos com $104 \mathrm{~g}$ a menos. Isto foi observado por Oliveira (1981) e Okada (1994) quando, mostrando a importância do manejo inicial, constataram que existe uma resposta positiva para o maior peso inicial dos pintos sobre o desempenho das aves. Da mesma forma que observado para o peso médio das aves, o consumo de ração (Tabela 3) foi afetado pelo peso inicial dos pintos até os 21 dias de idade $(\mathrm{P}<0,05)$, mas aos 35 e aos 42 dias não foi observada a mesma relação.

De 22 aos 35 dias de idade, os frangos provenientes dos pintos mais leves consumiram $212 \mathrm{~g}$ a menos, enquanto dos 36 aos 42 dias a diferença foi de apenas $3 \mathrm{~g}$, que indicando uma recuperação no consumo que 
Tabela 2 - Desempenho por fase de frangos de corte, criados com diferentes períodos de fornecimento de dieta préinicial

Table 2 - Performance per phase for broilers raised with different periods of pre-starter diets feeding

\begin{tabular}{|c|c|c|c|}
\hline & \multicolumn{3}{|c|}{$\begin{array}{l}\text { Período de fornecimento da dieta pré-inicial (dias) } \\
\text { Pre-starter diets feeding period (days) }\end{array}$} \\
\hline & $1-7 \mathrm{~d}$ & $1-10 \mathrm{~d}$ & $1-14 \mathrm{~d}$ \\
\hline & \multicolumn{3}{|c|}{$\begin{array}{l}\text { Peso vivo }(\mathrm{g}) \\
\text { Live weight }(\mathrm{g})\end{array}$} \\
\hline Peso aos 7 dias (live weight at 7 days of weight) & 152 & 149 & 151 \\
\hline Peso aos 10 dias (live weight at 10 days of weight) & 239 & 237 & 240 \\
\hline Peso aos 14 dias (live weight at 14 days of weight) & 381 & 376 & 374 \\
\hline Peso aos 21 dias (live weight at 21 days of weight) & 705 & 702 & 705 \\
\hline Peso aos 35 dias (live weight at 35 days of weight) & 1.418 & 1.456 & 1.462 \\
\hline \multirow{2}{*}{ Peso aos 42 dias (live weight at 42 days of weight) } & 1.870 & 1.870 & 1.905 \\
\hline & \multicolumn{3}{|c|}{$\begin{array}{l}\text { Consumo de ração }(\mathrm{g}) \\
\text { Feed intake }(g)\end{array}$} \\
\hline De 1 aos 7 dias (from 1 to 7 days of age) & 128 & 130 & 131 \\
\hline De 8 aos 10 dias (from 8 to 10 days of age) & 103 & 117 & 115 \\
\hline De 11 aos 14 dias (from 11 to 14 days of age) & 200 & 190 & 204 \\
\hline De 15 aos 21 dias (from 15 to 21 days of age) & 163 & 200 & 220 \\
\hline De 22 aos 35 dias (from 22 to 35 days of age) & 1.486 & 1.322 & 1.365 \\
\hline \multirow[t]{2}{*}{ De 36 aos 42 dias (from 36 to 42 days of age) } & 1.046 & 1.060 & 1.035 \\
\hline & \multicolumn{3}{|c|}{$\begin{array}{l}\text { Conversão alimentar } \\
\text { Feed-to-gain ratio }\end{array}$} \\
\hline De 1 aos 7 dias (from 1 to 7 days of age) & 1,16 & 1,23 & 1,19 \\
\hline De 8 aos 10 dias (from 8 to 10 days of age) & 1,80 & 1,33 & 1,31 \\
\hline De 11 aos 14 dias (from 11 to 14 days of age) & 1,41 & 1,46 & 1,52 \\
\hline De 15 aos 21 dias (from 15 to 21 days of age) & 1,40 & 1,56 & 1,57 \\
\hline De 22 aos 35 dias (from 22 to 35 days of age) & 2,08 & 1,92 & 1,96 \\
\hline De 36 aos 42 dias (from 36 to 42 days of age) & $2,32 \mathrm{~B}$ & $2,56 \mathrm{~A}$ & $2,35 \mathrm{~B}$ \\
\hline
\end{tabular}

Médias seguidas de letras diferentes diferem estatisticamente pelo teste Tukey $(P>0,05)$.

Means followed by different letters are statistically different by Tukey test $(P>.05)$.

não se refletiu em maior peso médio, como observado na Tabela 3. Quanto à conversão alimentar, os resultados indicam efeito do peso dos pintos apenas na primeira e na última semana, sendo respectivamente, inferior para aves acima de $40 \mathrm{~g}$ de 1 a 7 dias e superior no período de 35 a 42 dias de idade (Tabela 3).

Somente o consumo de ração no período total (Tabela 4) foi influenciado pelo peso inicial dos pintos, sendo que o período de fornecimento da dieta pré-inicial não teve qualquer efeito sobre os parâmetros de desempenho avaliados. Mesmo sem diferença estatística de peso ao final do experimento, os frangos provenientes de pintos mais pesados foram $104 \mathrm{~g}$ mais pesados do que os provenientes de pintos mais leves. Isso permite estimar que, para cada grama de diferença de peso do pinto no alojamento, corresponde a cerca de 9,4 gramas de diferença no peso final. Este resultado está próximo ao descrito por Okada (1994), que afirmou que pintos com peso inferior a $40 \mathrm{~g}$ são bastante suscetíveis a desafios sanitários, e acabam o período de criação com peso 10 a 15 gramas menor para cada grama de diferença no peso inicial.

Como já foi mencionado, o consumo de ração no período total foi influenciado pelo peso inicial dos pintos. Os frangos com peso inicial maior consumiram $331 \mathrm{~g}$ a mais que os mais leves. No trabalho de Araújo et al (1999) foi observado que o peso das frangos que receberam ração inicial foi inferior ao encontrado para frangos alimentados com dieta pré-inicial até os sete dias. Vieira \& Moran (1998a) verificaram que pintos provenientes de matrizes jovens ( 27 de idade) apresentaram desempenho pior quando comparados àqueles obtidos de matrizes mais velhas (65 semanas de idade), mas com melhor conversão alimentar (1,90 versus 1,93 , respectivamente), corroborando os resultados obtidos neste experimento. Nesse mesmo sentido, Rocha et al. (1999), em estudo sobre os níveis 
Tabela 3 - Desempenho por fase de frangos de corte, criados com diferentes pesos iniciais no primeiro dia Table 3 - Performance per phase for broilers raised with different one-day chicks weight

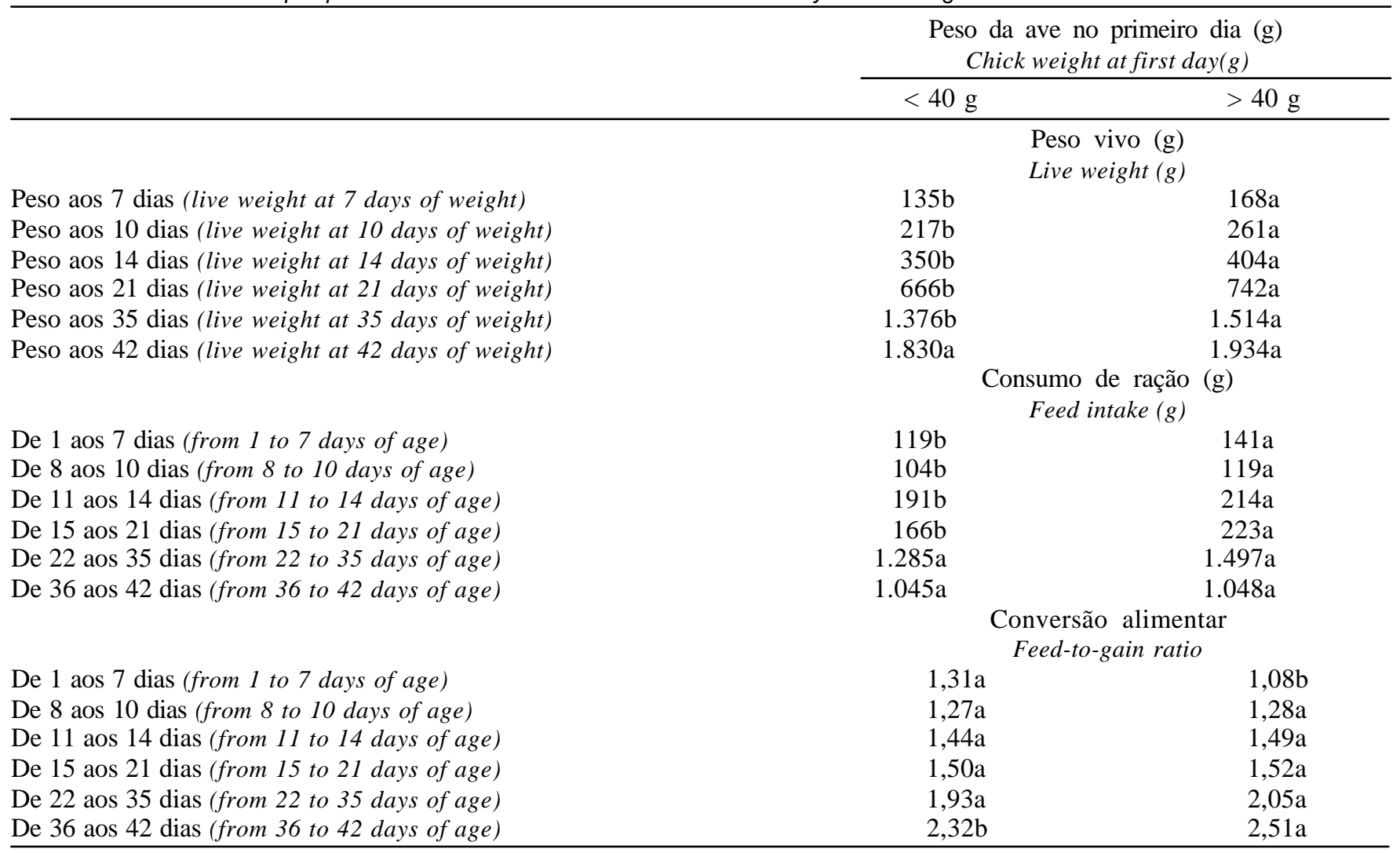

Médias seguidas de letras diferentes diferem estatisticamente pelo teste Tukey $(P>0,05)$.

Means followed by different letters are statistically different by Tukey test $(P>.05)$.

Tabela 4 - Desempenho de frangos de corte no período total, criados com diferentes períodos de fornecimento da ração pré-inicial e diferentes pesos iniciais

Table 4 - Performance of broilers raised with different periods of pre-starter diets and different one-day chicks weight

\begin{tabular}{|c|c|c|c|c|c|c|}
\hline \multirow[t]{2}{*}{$\begin{array}{l}\text { Período experimental } \\
\text { Experimental period }\end{array}$} & \multicolumn{2}{|c|}{$\begin{array}{l}\text { Peso da ave }(\mathrm{g}) \\
\text { Chick weight }(\mathrm{g})\end{array}$} & \multicolumn{3}{|c|}{$\begin{array}{c}\text { Fornecimento da ração pré-inicial (dias) } \\
\text { Pre-starter diets feeding period (days) }\end{array}$} & \multirow[t]{2}{*}{ CV (\%) } \\
\hline & $<40 \mathrm{~g}$ & $>40 \mathrm{~g}$ & $1-7$ & $1-10$ & $1-14$ & \\
\hline Peso médio (g) & 1.830 & 1.934 & 1.870 & 1.870 & 1.905 & 6,83 \\
\hline $\begin{array}{l}\text { Mean body weight }(g) \\
\text { Consumo de ração }(\mathrm{g}) \\
\text { Feed intake }(g)\end{array}$ & $2.994 b$ & $3.325 \mathrm{a}$ & 3.126 & 3.156 & 3.196 & 10,26 \\
\hline $\begin{array}{l}\text { Conversão alimentar } \\
\text { Feed-to-gain ratio }\end{array}$ & 1,67 & 1,75 & 1,70 & 1,72 & 1,71 & 6,11 \\
\hline $\begin{array}{l}\text { Viabilidade }(\%) \\
\text { Viability }(\%)\end{array}$ & 97,2 & 98,6 & 96,8 & 97,9 & 98,9 & 9,23 \\
\hline $\begin{array}{l}\text { Fator de produção } \\
\text { Production index }\end{array}$ & 248,5 & 253,9 & 247,0 & 249,4 & 257,2 & 10,33 \\
\hline
\end{tabular}

Médias seguidas de letras diferentes diferem estatisticamente pelo teste Tukey $(P>0,05)$.

Means followed by different letters are statistically different by Tukey test $(\mathrm{P}>$.05). 
de proteína bruta (20, 23 e 26\%) para frangos de 1 a 7 dias, observaram que o consumo de ração e a conversão alimentar foram influenciados pelos níveis empregados, e melhores para a dieta contendo $23 \%$ de proteína bruta.

Vieira (2001) alertou que as informações disponíveis na literatura são conflitantes quanto ao consumo de ração e conversão alimentar de pintos com diferentes pesos na eclosão e no alojamento, mas reitera que o peso corporal ao abate tem uma relação positiva com o peso do ovo e, por sua vez, com o peso do pintos ao nascer. Porém o desenvolvimento inicial é determinado por vários fatores, como tempo de jejum, dieta inicial, temperatura do ambiente nos primeiros dias de vida, dentre outros (Baião \& Aguilar, 2001; Gonzales \& Saldanha, 2001; Maiorka, 2001).

Os consumos de nutrientes e de energia, apresentados na Tabela 5, mostram que para as diferentes variáveis estudadas (proteína, energia metabolizável, metionina + cistina e lisina) não houve efeito significativo do programa de alimentação inicial. Todavia, o menor peso inicial do pinto, por acarretar menor consumo de ração, resultou em menores quantidades de nutrientes ingeridos. Quanto à energia metabolizável, é possível comparar com o trabalho efetuado por Maiorka et al. (1997), em que os autores constataram, para o período de até sete dias, uma semelhança nos valores de consumo para diferentes níveis de energia avaliados (2900, 3000 e $3100 \mathrm{kcal} / \mathrm{kg})$.

É de conhecimento que o peso dos órgãos na fase pré-inicial (Tabela 6) se constitui num fator de importância para caracterizar o bom desenvolvimento digestivo da ave. Baranyiová \& Holman (1976) constataram que o diâmetro dos intestinos delgado e grosso aumentaram com a idade, porém os autores não mediram as diferenças de peso.

Há diferenças comprovadas na comparação de linhagens de corte e de postura de 1 a 21 dias (Mahagna \& Nir, 1996) no que diz respeito ao desenvolvimento dos orgãos. Porém, apesar dos aumentos individuais de peso dos órgãos, Croom et al. (1999) afirmaram que a relação percentual do peso dos órgãos

Tabela 5 - Consumo de nutrientes de frangos de corte em diferentes idades, criados com diferentes períodos de fornecimento da ração pré-inicial e diferentes pesos iniciais

Table 5 - Nutrient intake of broilers raised at different periods of pre-starter diets and different one-day chicks weight

\begin{tabular}{|c|c|c|c|c|c|}
\hline \multirow[t]{2}{*}{$\begin{array}{l}\text { Período experimental } \\
\text { Experimental period }\end{array}$} & \multicolumn{2}{|c|}{$\begin{array}{l}\text { Peso da ave }(\mathrm{g}) \\
\text { Chick weight }(g)\end{array}$} & \multicolumn{3}{|c|}{$\begin{array}{l}\text { Fornecimento da ração pré-inicial (dias) } \\
\text { Pre-starter diets feeding period (days) }\end{array}$} \\
\hline & $<40 \mathrm{~g}$ & $>40 \mathrm{~g}$ & $1-7$ & $1-10$ & $1-14$ \\
\hline & \multicolumn{5}{|c|}{$\begin{array}{c}\text { Consumo de proteína }(\mathrm{g}) \\
\text { Protein intake }(g)\end{array}$} \\
\hline 1 a 7 dias & $26,1 \mathrm{~b}$ & $31,0 \mathrm{a}$ & 28,1 & 28,7 & 28,9 \\
\hline 1 a 10 dias & $48,4 \mathrm{~b}$ & $56,7 \mathrm{a}$ & 50,1 & 53,9 & 53,7 \\
\hline 1 a 14 dias & $88,5 \mathrm{~b}$ & $102,5 \mathrm{a}$ & 92,2 & 96,4 & 97,7 \\
\hline \multirow[t]{2}{*}{1 a 21 dias } & $123,3 \mathrm{~b}$ & $149,3 \mathrm{a}$ & 126,5 & 136,4 & 144,0 \\
\hline & \multicolumn{5}{|c|}{$\begin{array}{c}\text { Consumo de energia metabolizável (kcal) } \\
\text { Metabolizable energy intake (kcal) }\end{array}$} \\
\hline 1 a 7 dias & $350 \mathrm{~b}$ & $415 \mathrm{a}$ & 376 & 384 & 386 \\
\hline 1 a 10 dias & $659 \mathrm{~b}$ & $766 \mathrm{a}$ & 688 & 727 & 724 \\
\hline 1 a 14 dias & $1.231 \mathrm{~b}$ & $1.409 \mathrm{a}$ & 1.296 & 1.341 & 1.323 \\
\hline \multirow[t]{2}{*}{1 a 21 dias } & $6.257 \mathrm{~b}$ & $8.173 \mathrm{a}$ & 6.239 & 7.402 & 8.003 \\
\hline & \multicolumn{5}{|c|}{$\begin{array}{l}\text { Consumo de metionina }(\mathrm{g}) \\
\text { Methionine intake }(g)\end{array}$} \\
\hline 1 a 7 dias & $0,65 \mathrm{~b}$ & $0,78 \mathrm{a}$ & 0,70 & 0,72 & 0,72 \\
\hline 1 a 10 dias & $1,22 \mathrm{~b}$ & $1,42 \mathrm{a}$ & 1,25 & 1,36 & 1,36 \\
\hline 1 a 14 dias & $2,24 \mathrm{~b}$ & $2,57 \mathrm{a}$ & 2,31 & 2,43 & 2,48 \\
\hline \multirow[t]{2}{*}{1 a 21 dias } & $3,12 \mathrm{~b}$ & $3,75 \mathrm{a}$ & 3,17 & 3,49 & 3,65 \\
\hline & \multicolumn{5}{|c|}{$\begin{array}{c}\text { Consumo de lisina }(\mathrm{g}) \\
\text { Lysine intake }(\mathrm{g})\end{array}$} \\
\hline 1 a 7 dias & $1,40 \mathrm{~b}$ & $1,66 \mathrm{a}$ & 1,50 & 1,53 & 1,54 \\
\hline 1 a 10 dias & $2,60 \mathrm{~b}$ & $3,03 \mathrm{a}$ & 2,64 & 2,91 & 2,90 \\
\hline 1 a 14 dias & $4,75 \mathrm{~b}$ & $5,44 \mathrm{a}$ & 4,85 & 5,14 & 5,30 \\
\hline 1 a 21 dias & $6,58 \mathrm{~b}$ & $7,91 \mathrm{a}$ & 6,65 & 7,35 & 7,73 \\
\hline
\end{tabular}

Médias seguidas de letras diferentes diferem estatisticamente pelo teste Tukey $(P>0,05)$

Means followed by different letters are statistically different by Tukey test $(\mathrm{P}>.05)$. 
Tabela 6 - Medidas de relações corporais (comprimento do trato digestivo, pesos relativos de coração, pâncreas, fígado, baço e bursa de Fabricius) de frangos de corte aos 42 dias criados com diferentes idades de fornecimento da ração pré-inicial e com pintos com diferentes peso no primeiro dia de vida

Table 6 - Organ body percentual relations (gastrointestinal tract, heart, pancreas, liver and bursa) of broilers at 42 days of age raised with different periods of pre-starter diets feeding and different one-day chicks weight

\begin{tabular}{|c|c|c|c|}
\hline \multirow[t]{3}{*}{$\begin{array}{l}\text { Parâmetro } \\
\text { Parameter }\end{array}$} & \multicolumn{3}{|c|}{$\begin{array}{l}\text { Tratamento } \\
\text { Treatment }\end{array}$} \\
\hline & \multicolumn{3}{|c|}{$\begin{array}{l}\text { Fornecimento ração pré-inicial (dias) } \\
\text { Pre-starter diets feeding period (days) }\end{array}$} \\
\hline & $1-7$ & $1-10$ & $1-14$ \\
\hline Comprimento do trato digestivo $(\mathrm{cm})$ & 1,91 & 2,00 & 2,13 \\
\hline $\begin{array}{l}\text { Gastrointestinal tract length }(\mathrm{cm}) \\
\text { Peso relativo do coração }(\%) \\
\text { Heart relative weight }(\%)\end{array}$ & 0,469 & 0,584 & 0,586 \\
\hline $\begin{array}{l}\text { Peso relativo do pâncreas }(\%) \\
\text { Pancreas relative weight }(\%)\end{array}$ & 0,194 & 0,223 & 0,188 \\
\hline $\begin{array}{l}\text { Peso relativo do fígado }(\%) \\
\text { Liver relative weight }(\%)\end{array}$ & 1,752 & 2,055 & 1,711 \\
\hline $\begin{array}{l}\text { Peso relativo do baço }(\%) \\
\text { Slung relative weight }(\%)\end{array}$ & 0,128 & 0,145 & 0,114 \\
\hline $\begin{array}{l}\text { Peso relativo da bursa }(\%) \\
\text { Bursa relative weight }(\%)\end{array}$ & 0,102 & 0,138 & 0,216 \\
\hline
\end{tabular}

Bursa relative weight $(\%)$

Peso dos pintos no primeiro dia de vida $(\mathrm{g})$

Chicks weight at first day $(g)$

\begin{tabular}{|c|c|c|}
\hline & $\begin{array}{l}\text { Menos de } 40 \mathrm{~g} \\
\text { Less than } 40 \mathrm{~g}\end{array}$ & $\begin{array}{l}\text { Mais de } 40 \mathrm{~g} \\
\text { More than } 40 \mathrm{~g}\end{array}$ \\
\hline Comprimento trato digestivo $(\mathrm{cm})$ & 2,007 & 2,020 \\
\hline \multicolumn{3}{|l|}{ Gastrointestinal tract length $(\mathrm{cm})$} \\
\hline Peso relativo coração $(\%)$ & 0,540 & 0,553 \\
\hline \multicolumn{3}{|l|}{ Heart relative weight (\%) } \\
\hline Peso relativo pâncreas (\%) & 0,202 & 0,201 \\
\hline \multicolumn{3}{|l|}{ Pancreas relative weight (\%) } \\
\hline Peso relativo fígado $(\%)$ & 1,792 & 1,887 \\
\hline \multicolumn{3}{|l|}{ Liver relative weight (\%) } \\
\hline Peso relativo baço $(\%)$ & 0,126 & 0,132 \\
\hline \multicolumn{3}{|l|}{ Slung relative weight (\%) } \\
\hline Peso relativo bursa (\%) & 0,157 & 0,147 \\
\hline Bursa relative weight (\%) & & \\
\hline
\end{tabular}

Médias seguidas de letras diferentes diferem estatisticamente pelo teste Tukey $(P>0,05)$.

Means followed by different letters are statistically different by Tukey test $(P>.05)$.

digestivos reduz com a idade, como é observado para frangos e perus. No presente estudo os resultados das medidas das relações corporais entre os diferentes órgãos digestivos, coração, baço e bursa de Fabricius estão apresentados na Tabela 6. Observa-se que a dieta pré-inicial e o peso dos pintos de um dia não influenciaram nenhum dos parâmetros morfométricos estudados.

\section{Conclusões}

Como o desempenho e os dados morfométricos analisados não indicaram diferenças estatísticas significativas, concluiu-se que as idades de fornecimento da dieta pré-inicial (7, 10 ou 14 dias) não influenciaram, neste estudo, o desempenho de frangos de corte. Entretanto, quando o peso do pinto foi inferior a $40 \mathrm{~g}$, ocorreram redução significativa no consumo e tendência para pior peso dos frangos ao final do experimento. Os parâmetros morfométricos avaliados não foram afetados pelos tratamentos.

\section{Agradecimento}

Ao Abatedouro São Salvador e ao CNPq, pelo apoio financeiro e pela doação de materiais de consumo para a realização do experimento. 


\section{Literatura Citada}

ARAÚJO, C.S.S.; STRINGHINI, J.H.; ARAÚJO L.F. et al. Manejo nutricional de frangos de corte na fase pré-inicial. Archivos Latino-americanos de Producción Animal, v.7, n.2, p.77-84, 1999.

BAIÃO, N.C.; AGUILAR, C.A.L. Impacto do tempo de alojamento do pintinho de corte sobre a produção do frango. In: CONFERÊNCIA APINCO DE CIÊNCIA E TECNOLOGIA AVÍCOLAS, 2001, Campinas. Anais... Campinas: Fundação Apinco de Ciência e Tecnologia Avícolas, 2001. p.125140.

BARANYIOVÁ, E.; HOLMAN, J. Morphological changes in the intestinal wall in fed and fasted chickens in the first week after hatching. Acta Veterinaria Brno, v.45, p.151-158, 1976.

CASTRO A.G.M. Qualidade de pintos de um dia e importância do manejo no desempenho de frangos de corte. In: SIMPÓSIO GOIANO DE AVICULTURA, 2., 1996, Goiânia. Anais... Goiânia: Associação Goiana de Avicultura/UFG, 1996. p.67-70.

CROOM, W.J.; BRAKE, J.; COLES, B.A. et al. Is intestinal absorption capacity rate-limiting for performance in poultry. Journal of Applied Poultry Research, v. 8, n.2, p.242-252, 1999.

DIBNER, J. Nutritional requirements of young poultry. In: ARKANSAS NUTRITION CONFERENCE, 1996, Fayetteville. Proceedings...Fayetteville: Arkansas Poultry Federation, 1996. p.15-27.

FRANCO, S.G.; JUNQUEIRA, O.M.; COSTA-SILVA, G.J. et al. Programas de alimentação para frangos de corte. In: REUNIÃO DA SOCIEDADE BRASILEIRA DE ZOOTECNIA, 27., 1990, Campinas. Anais... Piracicaba: Sociedade Brasileira de Zootecnia, 1990. p.126.

FRANCO, S.G.; CURVELLO, F.A.; CAMPOS, E.J. et al. Programas alimentares para frangos de corte: I - Efeitos sobre o desempenho. Arquivos Brasileiros de Medicina Veterinária e Zootecnia, v.35, n.3, p.405-415, 1983.

GONZALES, E.; SALDAÑA, E.S.P.B. Os primeiros dias de vida do frango e a produtividade futura. In: CONGRESSO BRASILEIRO DE ZOOTECNIA, 11., 2001, Goiânia. Anais...Goiânia: Universidade Católica de Goiás, 2001. p.310-327.

MAHAGNA, M.; NIR, I. Comparative development of digestive organs, intestinal disaccharidases and some blood metabolites in broiler and layer-type chicks after hatching. British Poultry Science, v.37, n.3, p.359-371, 1996.

MAIORKA, A. Adaptações digestivas pós-eclosão. In: CONFERÊNCIA APINCO DE CIÊNCIA E TECNOLOGIA AVÍCOLAS, 2001, Campinas. Anais... Campinas: Fundação Apinco de Ciência e Tecnologia Avícolas, 2001. p.141-152.

MAIORKA, A.; LECZNIESKI, J.; BARTELS, H.A et al. Efeito do nível energético da ração sobre o desempenho de frangos de corte de 1 a 7,7 a 14, 14 a 21 dias de idade. In.: CONFERÊNCIA APINCO DE CIÊNCIA E TECNOLOGIA AVÍCOLAS, 1997, Campinas. Anais... Campinas: Fundação Apinco de Ciência e Tecnologia Avícolas, 1997. p.18.

MORAN, E.T. Digestion and absorption of carbohydrates in fowl and events through perinatal development. Journal of Nutrition, v.115, p.665-674, 1985.

NIR, I. Mecanismos de digestão e absorção de nutrientes durante. In: CONFERÊNCIA APINCO DE CIÊNCIA E TECNOLOGIA AVÍCOLAS, 1998, Campinas. Anais...
Campinas: Fundação Apinco de Ciência e Tecnologia Avícolas, 1998. p.81-91.

OKADA, T.M.A. Qualidade do pinto de um dia. In: PINHEIRO, M.R. (Ed.) Manejo de frangos. Campinas: Fundação Apinco de Ciência e Tecnologia Avícolas, 1994. p.41-46.

OLIVEIRA, R.L. Mortalidade inicial - causas e controle. In: CAMPOS E.J., LAMAS DA SILVA, J.M.; SILVA, E.N. (Eds.). Produção e qualidade de pintos de um dia Belo Horizonte, 1981. p.229-236.

PENZ JR., A.M.; VIEIRA S.L. Nutrição na primeira semana. In: CONFERÊNCIA APINCO DE CIÊNCIA E TECNOLOGIA AVÍCOLAS, 1998. Campinas. Anais...Campinas: Fundação Apinco de Ciência e Tecnologia Avícolas, 1998. p.121-39.

ROCHA, P.T.; ROBERT, N.; STRINGHINI, J.H. et al. Desempenho de frangos de corte criados com diferentes níveis de proteína bruta e energia metabolizável em rações pré-iniciais (1-7 dias). In: REUNIÃO ANUAL DA SOCIEDADE BRASILEIRA DE ZOOTECNIA, 36., 1999, Porto Alegre. Anais... Porto Alegre: Sociedade Brasileira de Zootecnia, 1999. p.184.

SERAFIN, J.A.; NESHEIM, M.C. Influence of dietary heatlabile factors in soybean meal upon bile acid pool and turnover in the chick. Journal of Nutrition, v.100, p.786796, 1970.

SHIROMA, N.N.; ZANETTI, M.A.; FARIA, D.E. et al. Efeito de programas alimentares sobre o desempenho de frangos de corte durante o inverno. In.: CONFERÊNCIA APINCO DE CIÊNCIA E TECNOLOGIA AVÍCOLAS, 1996, Campinas. Anais... Campinas: Fundação Apinco de Ciência e Tecnologia Avícolas. 1996. p.51.

TOLEDO, R.S.; VARGAS Jr., J.G.; ALBINO, L.F.T. et al. Aspectos práticos da nutrição pós-eclosão: níveis nutricionais utilizados, tipos de ingredientes e granulometria da dieta. In: CONFERÊNCIA APINCO DE CIÊNCIA E TECNOLOGIA AVÍCOLAS, 2001, Campinas. Anais... Campinas: Fundação Apinco de Ciência e Tecnologia Avícolas, 2001. p.153-167.

TRINDADE, D.S.; CAVALHEIRO, A.C.L.; OLIVEIRA M.F.G. et al. Efeito do nível de energia da dieta e do programa alimentar sobre o desempenho e composição química da carcaça de frangos para abate. Anuário Técnico do IPZFO, v.9, p.21-37, 1982.

VIEIRA, S.L. Idade da matriz, tamanho do ovo e desempenho do pintinho. In: CONFERÊNCIA APINCO DE CIÊNCIA E TECNOLOGIA AVÍCOLAS, 2001, Campinas. Anais...Campinas: Fundação Apinco de Ciência e Tecnologia Avícolas, 2001. p.117-123.

VIEIRA, S.L.; MORAN JR., E.T. Broiler yields using chicks from egg weight extremes in breeder age and dietary propionate. Journal of Applied Poultry Research, v.7, n.3, p.320-327, 1998a.

VIEIRA, S.L.; MORAN JR., E.T. Broiler yields using chicks from egg weight extremes and diverse strains. Journal of Applied Poultry Research, v.7, n.4, p.339-346, 1998 b.

ZÚNIGA, I.O.; CAMPOS, E.J.; FERREIRA, J.M. et al. Efeito de programas de alimentação sobre o desempenho de frangos de corte. Arquivos Brasileiros de Medicina Veterinária e Zootecnia v.46, n.6, p.675-683, 1994. 